
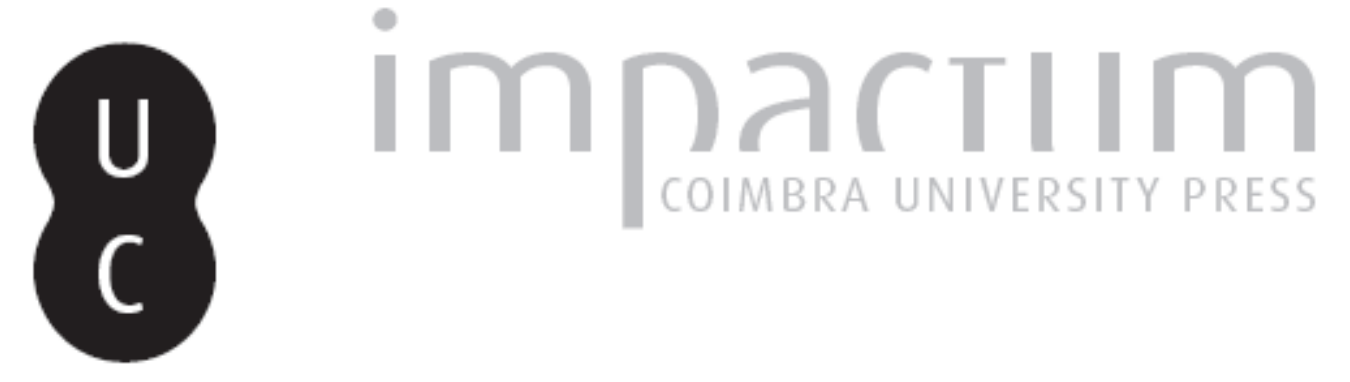

\title{
A segurança rodoviária e os acidentes: estudo de caso
}

Autor(es): Talaia, Mário; Amaro, Nuno

Publicado por: Associação Portuguesa de Riscos, Prevenção e Segurança

URL persistente:

URI:http://hdl.handle.net/10316.2/36022

DOI:

DOI:http://dx.doi.org/10.14195/1647-7723_19_23

Accessed : $\quad$ 26-Apr-2023 13:41:22

A navegação consulta e descarregamento dos títulos inseridos nas Bibliotecas Digitais UC Digitalis, UC Pombalina e UC Impactum, pressupõem a aceitação plena e sem reservas dos Termos e Condições de Uso destas Bibliotecas Digitais, disponíveis em https://digitalis.uc.pt/pt-pt/termos.

Conforme exposto nos referidos Termos e Condições de Uso, o descarregamento de títulos de acesso restrito requer uma licença válida de autorização devendo o utilizador aceder ao(s) documento(s) a partir de um endereço de IP da instituição detentora da supramencionada licença.

Ao utilizador é apenas permitido o descarregamento para uso pessoal, pelo que o emprego do(s) título(s) descarregado(s) para outro fim, designadamente comercial, carece de autorização do respetivo autor ou editor da obra.

Na medida em que todas as obras da UC Digitalis se encontram protegidas pelo Código do Direito de Autor e Direitos Conexos e demais legislação aplicável, toda a cópia, parcial ou total, deste documento, nos casos em que é legalmente admitida, deverá conter ou fazer-se acompanhar por este aviso.

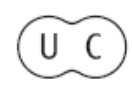




\section{territorium}

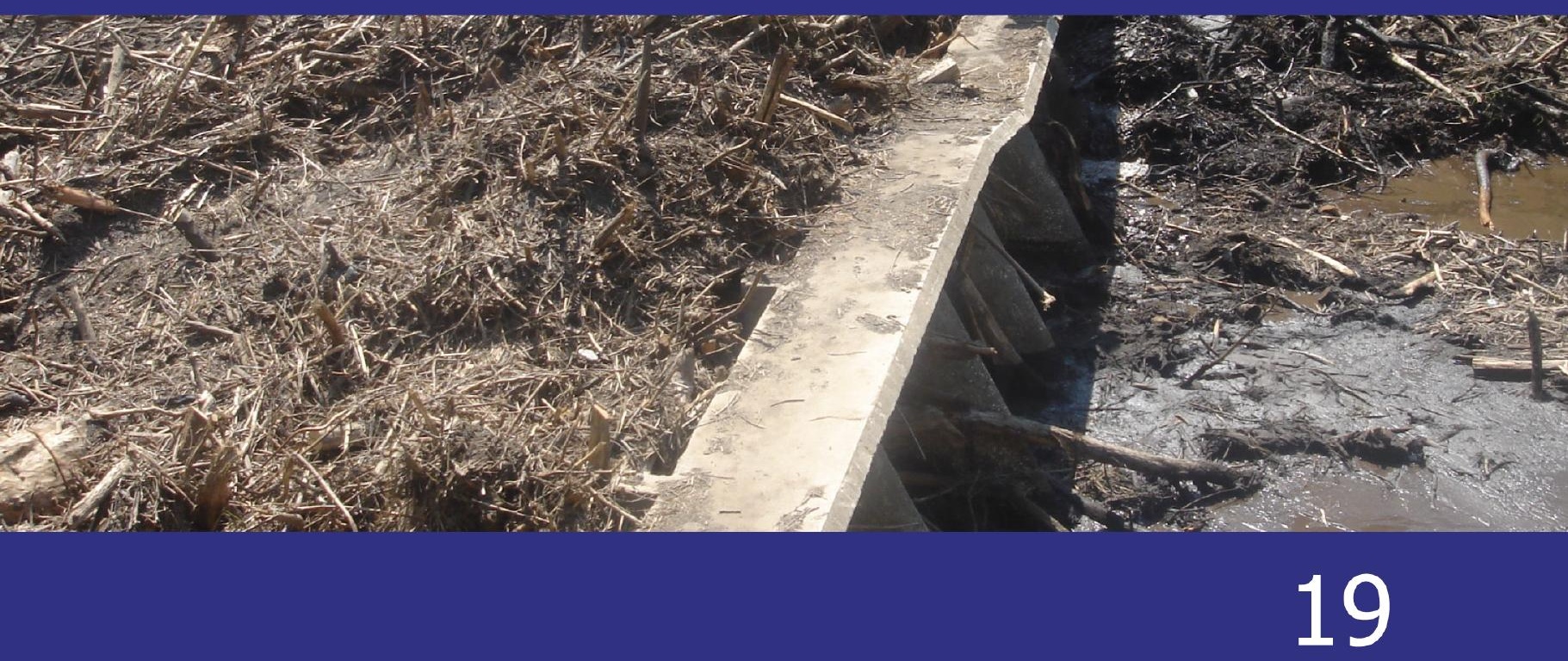

\section{Reequacionar o Conhecimento dos Riscos e das Catástrofes}

Revista da Associação Portuguesa de Riscos, Prevenção e Segurança 
Mário Talaia

Departamento de Física, Universidade de Aveiro, Campus Santiago, Aveiro mart@ua.pt

Nuno Amaro

ISCIA - Instituto Superior de Ciências da Informação e da Administração, Aveiro amaro.ntm@gmail.com

\section{RESUMO}

A segurança rodoviária deve interessar a todos os cidadãos. Todos podem contribuir para tornar as estradas mais seguras. Neste trabalho aborda-se um estudo de caso de um acidente, numa estrada plana e horizontal, envolvendo duas viaturas. 0 estudo mostra como a partir da localização da posição final das viaturas e das linhas de travagem é possível avaliar a velocidade na fase de percepção. Um algoritmo foi construído para simular variáveis de entrada no modelo. Foram também criados diferentes diagramas de resposta rápida.

Palavras chave: Segurança rodoviária, acidente de viação, atrito, princípio da conservação da energia, cidadania.

\section{RESUMEN}

Seguridad vial y accidentes: un estudio - La seguridad vial debe ser de interés para todos los ciudadanos. Es importante una segura circulación. En este trabajo se presenta un ejemplo de un accidente en una carretera plana y horizontal, con dos vehículos. El estudio muestra que los registros de la posición final de los vehículos y líneas de frenado permiten determinar diferentes velocidades. Un algoritmo se construyó para simular resultados. Diagramas se presentan para crear una respuesta rápida.

Palabras clave: Seguridad vial, accidentes de tráfico, fricción, ley de la conservación de la energía, ciudadanía.

\section{RÉSUMÉ}

Sécurité routière et accidents: étude de cas - La sécurité routière doit intéresser tous les citoyens. Tous peuvent contribuer pour rendre les routes plus sûres. Dans ce travail, on aborde l'étude de cas d'un accident, sur une route plane et horizontale, entre deux véhicules. L'étude démontre comme il est possible, à partir de la localisation de la position finale des véhicules et des lignes de freinage, évaluer la vitesse au cours de la phase de perception. Un algorithme a été créé pour simuler des variables d'entrée dans le modèle. Différents diagrammes de réponse rapide ont également été créés.

Mots clés: Sécurité routière, accidents, frottement, loi de la conservation de l'énergie, citoyenneté.

\section{ABSTRACT}

Road safety and accident: case study - Road safety should be of interest to all citizens. Anyone can contribute to make roads safer. In this paper, we will broach an accident case study on a flat and horizontal road, involving two vehicles. The study shows how to, from the vehicles final position location and braking lines, evaluate the speed during the moment of perception. To simulate the input variables in the model, an algorithm was created. Different rapid response diagrams were also created.

Key words: Road safety, accident, friction, law of conservation of energy, citizenship.

* O texto deste artigo corresponde à comunicação apresentada ao II Congresso Internacional de Riscos e VI Encontro Nacional, tendo sido submetido para revisão em 10-06-2010, tendo sido aceite para publicação em 21-07-2010. Este artigo é parte integrante da Revista Territorium, n. ${ }^{\circ} 19,2012,{ }^{\circ}$ RIscos, ISBN: 0872- 8941 


\section{Introdução}

A segurança rodoviária interessa a todos os cidadãos. É responsabilidade de cada um desenvolver atributos para tornar as estradas mais seguras. Embora, a eficácia das acções já realizadas, na União Europeia (UE), em particular, o número de vítimas das estradas continua a ser considerado demasiado elevado.

O comportamento dos condutores é, reconhecidamente, a primeira causa dos acidentes mortais, sendo a velocidade considerada como factor determinante. Este problema tem sido alvo de atenção crescente em toda a UE, tendo sido proposta uma meta ambiciosa, ou seja reduzir em cerca de 50\% o número de vítimas até 2010 em relação a 2001. Para alcançar esta meta, é necessária uma abordagem sistemática (R.Gomes, 2005).

Um acidente pode ser considerado um acontecimento fortuito ou eventual que altera a ordem das coisas, que involuntariamente pode originar danos às pessoas ou objectos, que se produz numa via aberta à circulação pública, que por causa do mesmo, uma ou várias pessoas resultem mortas ou feridas ou se produzam danos materiais e que esteja implicado pelo menos um veículo.

Há diferentes factores que podem influenciar um acidente. Poder-se-á afirmar que, a causa de um acidente é qualquer comportamento, condição, acto ou negligência sem o qual este não se teria produzido.

O acidente rodoviário ocorre quando se verifica uma ruptura entre os diversos componentes do trânsito, ou seja entre o utente, o veículo e o ambiente. No geral, é aceite que a ocorrência de um acidente inclui três fases, nomeadamente a fase de percepção, a fase de decisão e a fase de conflito

\section{Teoria}

É sabido que um acidente, apesar da sua rapidez, não acontece de forma instantânea, mas é uma situação que sofre uma evolução que se desenvolve no tempo e no espaço, mediante uma série de circunstâncias sucessivas que se vão produzindo até ao surgimento do resultado.

De acordo com a Direcção Geral de Viação (D.G.V., 1998), acidente é “ocorrência na via pública ou que nela tenha origem envolvendo pelo menos um veiculo do conhecimento das entidades fiscalizadoras da qual resultem vitimas e / ou danos materiais". No geral, pode afirmar-se que a causa de um acidente é qualquer comportamento, condição, acto ou negligência sem o qual este não se teria produzido (J. BAKER e L. FRICKE, 1998 e 1990; L. Fricke, 1990).

Do ponto de vista de uma classificação clássica é possível distinguir três fases: Fase de Percepção (FP), Fase de Decisão (FD) e Fase de Conflito (FC).
Considere-se, agora, alguns aspectos inerentes a cada uma das três fases que são aceites e consideradas neste trabalho de investigação.

A Fase de Percepção ( $1^{\text {a }}$ fase de intervenção) está delimitada pelo Ponto de Percepção Possível (PPP) e pelo Ponto de Decisão (PD). Pode ser considerada uma fase ampla do acidente devido ao condutor ou peão compreender todo o processo no qual se vê implicado.

De notar que o Ponto de Percepção Real (PPR) ocorre normalmente a seguir ao PPP e sempre antes do PD. Nestes termos, o PPP tem a ver com o momento e lugar onde o movimento ou a situação inesperada foi percebida pelo indivíduo e ocorre antes ou no momento exacto da percepção real do facto. O PPR tem a ver com o momento e lugar no qual o condutor ou peão percebeu realmente pela primeira vez o perigo ou a situação anormal e, na prática, sempre posterior ao PPP (no limite pode-se assumir como coincidente).

Assim, numa investigação a fixação do PPR é de extrema importância, pois este pode ser condicionado por factores físicos ou psíquicos do indivíduo que podem ter influenciado a avaliação de seus actos.

No entanto, há situações em que o PPR pode não existir ou estar muito próximo do Ponto de Conflito (PC) e que não seja possível fazer a sua distinção. O PC é o ponto no qual o acidente se dá e corresponde à posição de máximo efeito.

Por outras palavras, o condutor após o PPR deve reagir ao estímulo de intervenção decidindo que estratégia deve adoptar, fazendo este processo parte da FD e da área de percepção. No geral, esta área engloba a área de manobra, a qual compreende as fases de decisão e conflito, sequenciais à fase de percepção. Ou seja, compreende todo o processo de acidente desde o PPP até ao Ponto Final (PF). O PF refere-se à posição final e relata a posição dos veículos e objectos quando se imobilizam. Há situações em que o PC coincide com o PF.

A Fase de Decisão ( $2^{\mathrm{a}}$ fase de intervenção) pode ser anulada pela rapidez dos acontecimentos, chegando nalguns casos a não existir. Nestas circunstâncias, ocorre apenas a percepção e os resultados. A FD está delimitada pelo PD e pelo Ponto Chave (PCH). O PCH é o ponto no qual o acidente é inevitável. Pode coincidir com o PPR quando não é possível manobra evasiva ou quando esta é muito reduzida. Nesta fase está incluído o tempo que demora o condutor a compreender a situação, tomar uma decisão e efectuar a manobra de evasão. Assim sendo, desde que o condutor ou peão se aperceba do perigo até actuar demora algum tempo. Este tempo denominado de Tempo de Reacção (TR) valoriza a chegada de estímulos exteriores, o processamento de informação e prepara medidas de intervenção e, a resposta - solução para agir. 
O TR depende da idade, do estado físico e psíquico de cada indivíduo. É senso comum, que o avanço da idade, o cansaço e a desatenção aumentam o TR. O seu valor típico pode oscilar entre $0,4 \mathrm{~s}$ e $2,0 \mathrm{~s}$.

A Fase de Conflito ( $3^{\mathrm{a}}$ fase de intervenção) é o culminar do acidente, ou seja, compreende a última fase da evolução do acidente.

Nesta fase a área de conflito é a área onde se verifica a possibilidade de ocorrer o acidente. É uma área variável, pois depende da trajectória dos veículos, dos elementos intervenientes e da acção evasiva. A área de conflito pode coincidir com a área de manobra (inclui a área de decisão e área de conflito).

Com base na distância entre o PPP e o PPR é possível divagar acerca de uma investigação psíquica e somática de agentes intervenientes e de condições ambientais. Por exemplo, para uma distância com algum significado ou valorizada, é possível reflectir com grande probabilidade de acerto, que o condutor ia distraído por qualquer motivo, condicionado por sono, cansaço, álcool, medicamentos, doença, conversa, distracção e outros.

Com base na distância entre o PPR e o PD é possível divagar acerca de uma investigação somática e psíquica do indivíduo centrando-se nos seus conhecimentos experiência e reflexos (medição do tempo de reacção). A uma distância significativa pode corresponder uma reacção lenta, pouco enérgica baseada numa lenta tomada de decisão ou na sua execução, ou seja o indivíduo teve um fraco processamento para adoptar estratégias de intervenção. Nesta distância a distracção não deve ser valorizada, pois esta é considerada na distância entre o PPP e o PPR.

As manobras de evasão, com o objectivo de evitar um resultado danoso ou diminuir as suas consequências, estão incluídas entre o PD e PCH. Nestas circunstâncias deve ser valorizada a perícia do condutor e seus conhecimentos sobre acções evasivas (devem ser considerados registos anteriores) assim como o estado de funcionamento dos principais órgãos do veículo (eficiência de resposta à solicitação do condutor).

A cinemática e a dinâmica ajudam a determinar valores de velocidade, de tempo e de distância entre cada ponto (R. Serway e J. Jewett, 2004; D. Halliday e R. RESNICK, 2005).

Como o atrito é de extrema importância na estimativa de valores recorreu-se também ao princípio da conservação da energia para um pavimento plano e horizontal.

Como já referido, este trabalho considera um estudo de caso em que é registada uma trajectória rectilínea.

A velocidade de um móvel, num movimento uniforme, é dada pela expressão em que d representa a distância percorrida e t o tempo necessário para percorrer essa distância.

$$
v=\frac{d}{t}
$$

Num movimento uniformemente variado a expressão será

$$
v_{f}=v_{i}+a t
$$

em que $V_{f}$ representa a velocidade final, $V_{i}$ a velocidade inicial, a a aceleração ou desaceleração e t o tempo.

A distância percorrida quando o veículo tem um movimento uniformemente variado é determinada a partir da integração da expressão (2). Assim,

$$
d=v_{i} t+\frac{1}{2} a t^{2}
$$

A expressão (2) pode ser reescrita,

$$
a=\frac{V_{f}-V_{i}}{t}
$$

Se a expressão (4) for introduzida na expressão (3), usando manipulações matemáticas, obtém-se para valor da distância percorrida

$$
d=\frac{V_{f}+V_{i}}{2} t
$$

Também, a variação da energia cinética, ou seja a diferença entre a energia cinética inicial e energia cinética final, é dada por

$$
\Delta \mathrm{E}_{\mathrm{c}}=\frac{1}{2} \mathrm{mv}_{i}^{2}-\frac{1}{2} \mathrm{mv}_{\mathrm{f}}^{2}
$$

Durante a travagem (com marca no pavimento), a variação da energia de dissipação permite escrever

$$
\Delta E_{a}=F d \Leftrightarrow \Delta E_{a}=\mu m g d
$$

em que $\mu$ representa o coeficiente de atrito entre duas superfícies, $m$ a massa do veículo, $d$ a distância de travagem e $\mathrm{g}$ a aceleração da gravidade.

Aplicando o princípio da conservação de energia, para o caso particular em estudo e assumindo algumas aproximações, pode-se escrever

$$
\Delta \mathrm{E}_{c}=\Delta \mathrm{E}_{a}
$$

Se as expressões (6) e (7) forem introduzidas na igualdade (8) obtém-se

$$
v_{i}=\sqrt{2 \mu g d+v_{f}^{2}}
$$


Para o caso particular da velocidade final ser considerada nula a expressão (9) toma o aspecto

$$
v_{i}=\sqrt{2 \mu g d}
$$

Conhecida a distância de travagem e assumindo um valor para o coeficiente de atrito é possível determinar a velocidade inicial usando a expressão (10), no trajecto que se está a considerar (situação entre pontos).

No caso de estudo, considerou-se PPP coincidente com PPR. Entre PPR e PD considerou-se um movimento uniforme assumindo um tempo de reacção para o indivíduo (valor típico, entre 0,4s a 2,0s); entre PD e PBRs (Ponto de Bloqueio das Rodas sem marcas) considerou-se um movimento uniformemente variado (deve ser assumido um tempo de resposta do sistema de travagem, normalmente de $0,2 \mathrm{~s}$ a $0,6 \mathrm{~s}$ ); entre PRBs e PBRc (Ponto de Bloqueio das Rodas com marcas) assume-se uma distância de $1 \mathrm{~m}$ a $4 \mathrm{~m}$. Normalmente é aceite $1 \mathrm{~m}$; entre PBRc e PF considerou-se movimento uniformemente variado.

A partir das expressões de (1) a (10) é possível desenhar toda a evolução do acidente em termos de cenário. 0 algoritmo construído, a partir de dados de entrada como a distância de travagem, coeficiente de atrito, tempo de reacção, tempo de resposta do sistema de travagem, distância entre o ponto de bloqueio das rodas sem e com marcas, permite obter em cada situação entre pontos, distâncias parciais, distância total, tempos parciais, tempo total e velocidades iniciais e velocidades finais entre pontos. O culminar do algoritmo indica o valor da velocidade no PPR.

Os diagramas construídos permitem para valores diferentes de atrito fazer uma estimativa da velocidade inicial (entre dois valores, máximo e mínimo) no PPP ou PPR, o tempo gasto durante todo o processo e distância envolvida.

É de realçar que os valores avaliados devem ser entendidos como orientadores para a interpretação do processo de evolução do acidente.

\section{Resultados e discussão}

O algoritmo considerou as leis da cinemática e de dinâmica. A figura 1 mostra a folha de rosto para um caso em que a distância de travagem é de 56,0m.

Como se mostra na figura 1, no cabeçalho da página é indicado que PPP coincidente com PPR.

O analista pode introduzir dados de entrada, tais como registos efectuados no pavimento, coeficiente de atrito que é função das condições atmosféricas e da qualidade do pavimento e pneus, distância entre os
PBR sem e com marcas no pavimento (valores típicos podem situar-se entre 1,0m e 4,0m (a experiência do analista é determinante), tempo de reacção do indivíduo (o tempo é influenciado por diferentes factores, no entanto, valores típicos aceites variam entre $0,2 \mathrm{~s}$ e $2,0 \mathrm{~s}$ ) e tempo de resposta do sistema de travagem que pode variar entre $0,2 \mathrm{~s}$ e $0,6 \mathrm{~s}$ (neste aspecto, a experiência do analista é determinante).

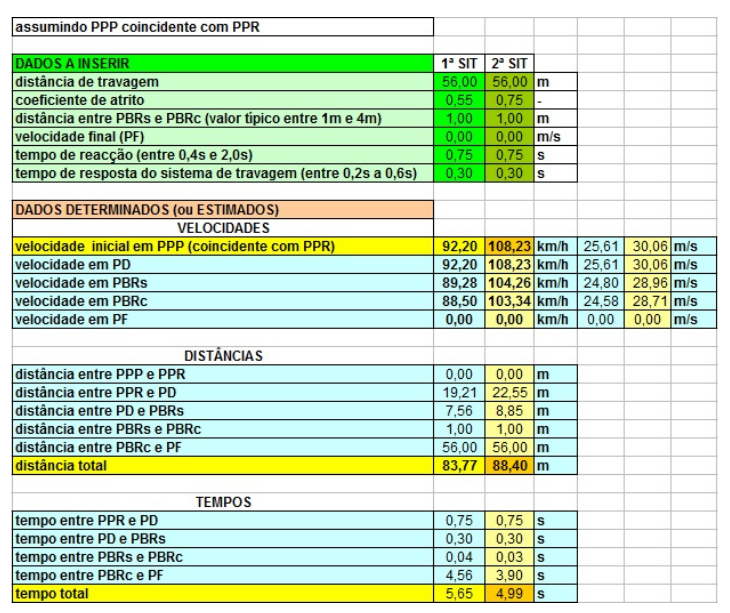

Fig. 1 - Folha de rosto para a avaliação da evolução de um acidente.

O analista pode variar cada um dos parâmetros anteriormente citados, mas deve, sempre considerar, duas possibilidades de coeficiente de atrito (por ser um valor com alguma subjectividade). 0 coeficiente de atrito depende das superfícies em contacto, do estado do pavimento e das condições atmosféricas. 0 valor a adoptar pode variar entre 0,5 e 0,9. A experiência do analista é muito importante para considerar sempre um valor mínimo e máximo. Laboratórios podem dar alguma ajuda acerca de valores adequados para o coeficiente de atrito (J. Winson e C. Hermandez-Hall, 1998; C. Bernard e C. Epp, 1995).

$\mathrm{Na}$ folha de rosto aparece a informação $1^{\text {a }}$ SIT e $2^{\mathrm{a}}$ SIT que significa primeira e segunda situação, respectivamente. No que concerne aos valores determinados ou estimados de velocidade, a folha de rosto, mostra para as duas situações consideradas a velocidade inicial do veículo em PPP (coincidente com PPR), a velocidade em PP, a velocidade em PBRs, a velocidade em PBRc e a velocidade em PF. A velocidade inicial em PPP (coincidente com PPR) está num fundo amarelo por ser muito importante para a avaliação da evolução do acidente.

O algoritmo também mostra o tempo total gasto e distância total num fundo amarelo, ambos entre PPP ou PPR e PF por serem duas informações muito importantes para o analista. No entanto, para o esclarecimento de dados, a folha de rosto mostra tempos parciais e distâncias parciais, entre pontos. 
Para distâncias parciais são visualizadas as seguintes distâncias: entre PPP e PPR (nesta situação nula); entre PPR e PBRs; entre PBRs e PBRc; entre PBRc e PF. Para tempos parciais são visualizados os seguintes tempos: entre PPP e PPR (nesta situação nulo); entre PPR e PBRs; entre PBRs e PBRc; entre PBRc e PF.

0 analista pode variar em qualquer momento um parâmetro mantendo outros, nas zonas marcadas a verde. Nestas circunstâncias pode avaliar as incertezas para a simulação e para as variáveis procuradas (velocidade, distância e tempo).

O algoritmo complementa a informação traçando diferentes diagramas de informação e localizando o caso investigado.

Na figura 2 mostram-se duas linhas que indicam como a distância de travagem influencia a velocidade do veículo no PPP ou PPR. A linha de cor "azul" considera um coeficiente de atrito de 0,75 e a linha cor de "tijolo" considera um coeficiente de 0,55. Em ambos os casos a velocidade final foi assumida ser nula. Na figura 2 são mostrados dois círculos, um de cor "azulado" e outro de "dourado" que representam a localização de um valor mínimo e máximo para a velocidade do veículo no PPP ou PPR. Como seria esperado, quando se mantêm todos os parâmetros de entrada e se aumenta o coeficiente de atrito a velocidade em PPP ou PPR. também aumenta.

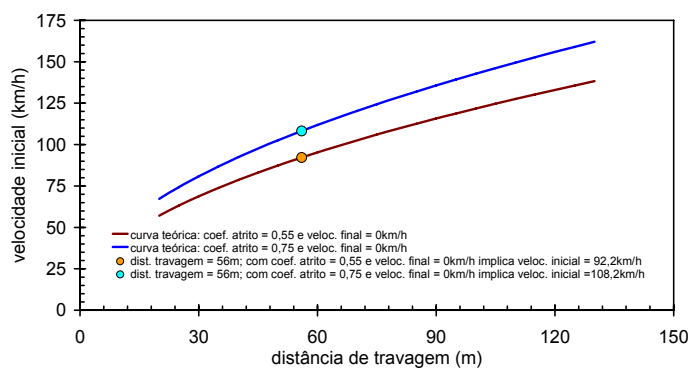

Fig. 2 - Folha de rosto: influência da distância de travagem na velocidade do veículo em PPP ou PPR.

$\mathrm{Na}$ figura 3 as duas linhas indicadas mostram como a distância de travagem influencia o tempo decorrido desde o momento que o condutor observa o obstáculo e a posição final, ou seja o tempo total durante a evolução do acidente. Cada linha é projectada função de um coeficiente de atrito, como se indica na figura. Os círculos mostram os tempos para cada situação avaliada. Como seria esperado, quando se mantêm todos os parâmetros de entrada e se aumenta o coeficiente de atrito o tempo total inerente à evolução do acidente diminui.

Também, na figura 4 as duas linhas mostram como a distância de travagem influencia a distância total desde o momento que o condutor observa o obstáculo e a posição final, ou seja a distância total durante a evolução do acidente. Cada linha é projectada função do coeficiente de atrito considerado e indicado na figura. Os círculos mostram as distâncias totais para cada situação avaliada. Como seria esperado, quando se mantêm todos os parâmetros de entrada e se aumenta o coeficiente de atrito a distância total inerente à evolução do acidente aumenta.

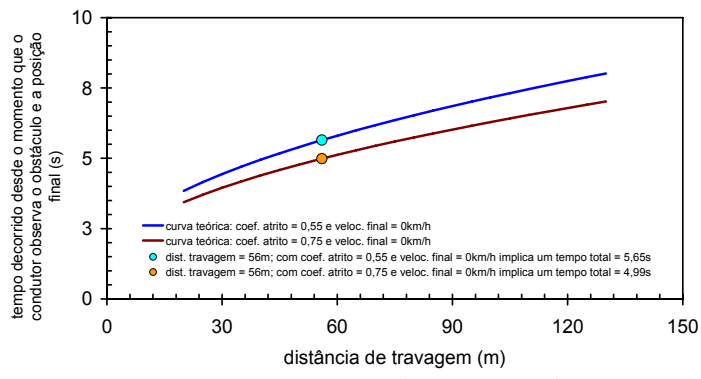

Fig. 3 - Folha de rosto: influência da distância de travagem no tempo total (entre PPP ou PPR e PF).

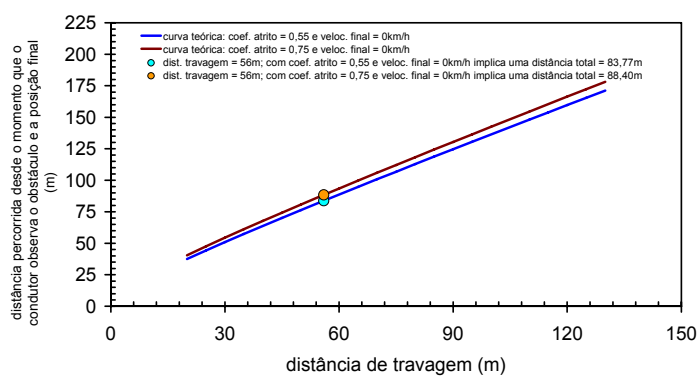

Fig. 4 - Folha de rosto: influência da distância de travagem na distância total (entre PPP ou PPR e PF).

A figura 5 é muito interessante por permitir uma visualização muito completa da evolução do acidente e de toda a descrição feita anteriormente.

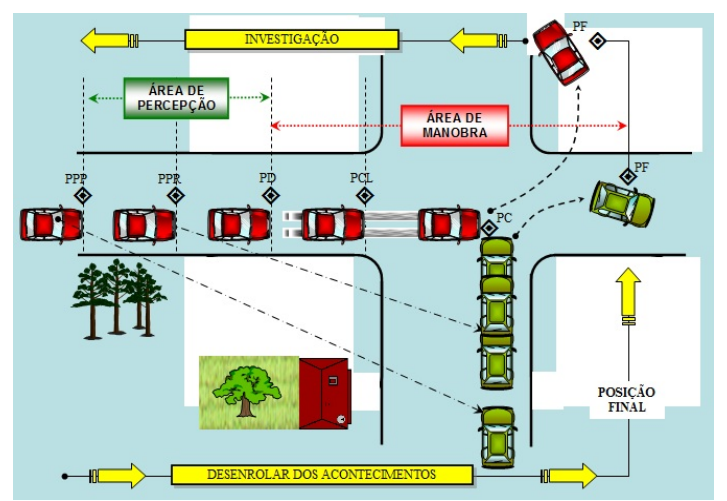

Fig. 5 - Desenvolvimento dos acontecimentos de uma investigação de um acidente.

\section{Considerações finais}

Neste trabalho, o desenvolvimento dos acontecimentos de uma investigação de um acidente foi considerado.

Um algoritmo foi desenvolvido de modo a permitir avaliar a velocidade de um veículo no PPP ou PPR, a distância total entre o PPP ou PPR e PF e o tempo total dispendido entre o PPP ou PPR e PF. 
A zona verde do algoritmo permite a partir da entrada de vários parâmetros de entrada, que podem ser considerados de alguma subjectividade, conhecer a incerteza de cada parâmetro de saída, nomeadamente o que estão em fundo "amarelo", ou seja a velocidade no PPR, a distância total entre PPR e PF e o tempo total dispendido entre PPR e PF.

A incerteza obtida para a velocidade no PPP ou PPR contribuirá para avaliar a evolução do acidente.

A vantagem do algoritmo desenvolvido é facilitar a visualização imediata de parâmetros de análise de acidente e anular qualquer erro de contas através do uso de máquinas de calcular.

Os diagramas complementares permitem uma abordagem visual da evolução do acidente, considerando os dados de entrada e a estimativa de resultados.

\section{Referências Bibliográficas}

BAKER, J.S. e FrICKE, L.B. (1998) - "The Traffic-Accident Investigation Manual: At-Scene Investigation and Technical Follow-Up". 9th Edition. ISBN10: 0912642068. Northwestern University Traffic Institute.

BAKer, J.S. e FrICKE, L.B. (1990) - "The Traffic-Accident Investigation Manual". Volume II. Northwestern University Traffic Institute.
Bernard, C.H. e EPP, C.D. (1995) - Laboratory Experiments in College Physics. John Wiley \& Sons. Inc.

D.G.V. (1998) - "Plano Integrado de Segurança Rodoviária. Direcção Geral de Viação". Lisboa.

GOMES, R.M. (2005) - "Educação Rodoviária: segurança e promoção da saúde como estratégias de uma cidadania consciente". MCEC. Saúde e o grande público. (http://www2.dce.ua.pt/ funcionarios/gomes/ documentos/educacao_ rodoviaria.pdf).

FRICKE, L.B. (1990) - "Traffic Accident Reconstruction. Traffic-Accident Investigation Manual". Northwestern University, Evanston Editors. ISBN: 0-912642-07-6.

Halliday, D. e Resnick, R. (2005) - "Fundamentals of Physics". 7th Edition. Rensselaer Polytechnic Institute, Jerald Walker, Cleveland State Univ. ISBN: 978-0-471-21643-8, 1136 pages.

Serway, R.A. e JeWett, J.W. (2004) - "Physics for Scientists and Engineers". 6th Edition, ISBN: 0534408427.

Winson, J.D. e Hermandez-Hall, C.A. (1998) - "Physics Laboratory Experiments". Houghton Mifflin Company, USA. 